\title{
Lidil
}

Revue de linguistique et de didactique des langues

40 | 2009

La motivation pour l'apprentissage d'une langue seconde

\section{Apprentissage du français et motivation existentielle. Le cas des universités à Taiwan}

\section{Serge Dreyer}

\section{(2) OpenEdition}

\section{Journals}

Édition électronique

URL : http://journals.openedition.org/lidil/2911

DOI : 10.4000/lidil.2911

ISSN : 1960-6052

Éditeur

UGA Éditions/Université Grenoble Alpes

Édition imprimée

Date de publication : 1 décembre 2009

Pagination : 31-47

ISBN : 978-2-84310-154-0

ISSN : $1146-6480$

Référence électronique

Serge Dreyer, «Apprentissage du français et motivation existentielle. Le cas des universités à Taiwan », Lidil [En ligne], 40 | 2009, mis en ligne le 01 juin 2011, consulté le 19 avril 2019. URL : http:// journals.openedition.org/lidil/2911; DOI : 10.4000/lidil.2911

(c) Lidil 


\title{
Apprentissage du français et motivation existentielle. Le cas des universités à Taiwan
}

\author{
Serge Dreyer*
}

RÉSUMÉ

En didactique du français langue étrangère (FLE), la notion de motivation pour l'apprentissage de la langue qui fait l'objet du plus large consensus parmi les enseignants-chercheurs fait référence à la théorie de la motivation instrumentale / intégrative (Gardner et Lambert, 1972). Cette théorie, qui reste valide pour de nombreuses situations, ne répond toutefois pas à celle de nos apprenants du français en langue optionnelle dans les universités de Taiwan. La plupart d'entre eux, en choisissant le français, ne mentionnent ni des attentes sur le plan professionnel (ou bien de manière très vague), ni une perspective d'intégration dans une population francophone.

Nous montrerons dans cet article que leur motivation est à relier au contexte sociétal dans lequel ils évoluent et qu'elle est étroitement associée à une démarche identitaire dans laquelle les représentations sociales jouent un rôle important. C'est la raison pour laquelle nous appellerons ce type de motivation existentielle.

\section{ABSTRACT}

In the field of the didactics of French as a Foreign Language (FLE in French), the concept of motivation in the process of learning French which is widely accepted among teachers and researchers refers to the theory of instrumental / integrative motivation (Gardner and Lambert, 1972). This theory, which remains valid for many educative situations, does not characterize most learners' motivation who chose French as an optional language in their curriculum in Taiwanese universities. When asked about the reason why they chose French, they usually do not refer to any anticipated professional benefit nor do they plan any integration in a French speaking community.

We will explain in this article that their motivation should be understood in reference to the change of the society in which they live and

* Professeur associé de français, Université Tunghai, Taichung, Taiwan. 
that a quest for their identity is closely associated with this motivation in which social representation plays a very important role.

L'absence relative de motivation des apprenants pour l'apprentissage du français dans la zone asiatique non francophone est devenue le sujet fréquent des propos des enseignants et des chercheurs (Tang, 2004 ; Bok, 2004). Dans ce topos, on trouve généralement sur le banc des accusés responsables du déclin de l'étude de la langue française, la prépondérance de l'apprentissage de l'anglais, la faible présence économique et politique de la France et le manque de perspectives professionnalisantes. Les difficultés d'ordre linguistique que connaissent les apprenants asiatiques dans leur apprentissage du français ne feraient qu'aggraver la situation. Ce pessimisme ambiant nous a conduit à chercher localement une solution partielle pour enrayer ce déclin du choix du cours de français. Selon nous, la prise en compte des dimensions culturelles de l'enseignement du français répond d'une part aux aspirations explicites de nos apprenants, et d'autre part, elle permet de satisfaire à leur besoin d'affirmation identitaire qui jusqu'à présent ne faisait pas l'objet d'une quelconque analyse dans la recherche en didactique du français à Taiwan. Nous avons donc élaboré un nouveau concept que nous appelons «motivation existentielle » et que nous pensons opératoire pour de nombreuses situations de l'enseignement du français en Asie $^{1}$. Ce concept fait appel en particulier aux notions de représentation sociale, de valeur, d'identité et prend en compte l'environnement socioéconomique et politique.

\section{Question de motivation}

Notre public est constitué d'étudiants provenant de diverses filières qui choisissent le cours de français qui est proposé en cours optionnel (payant) sur trois ans, à raison de 3 heures par semaine, dans le cadre d'un département d'anglais. Ce cours est dispensé sur deux semestres, ce qui représente environ 80 heures. Il se trouve en concurrence directe avec des cours d'allemand, d'espagnol, de japonais, d'anglais pour les langues étrangères. Mais nombre d'autres matières optionnelles peu-

1. Chi Lee, Discours d'ouverture du $1^{\text {er }}$ Congrès régional Asie-Pacifique de la Fédération internationale des professeurs de français, Taipei, Taiwan, 27/04 au $01 / 05 / 2006$. 
vent être choisies par les étudiants pour satisfaire aux exigences institutionnelles. On évolue donc dans un véritable marché de l'éducation, ce qui rend encore plus pressante la nécessité de cerner la motivation des apprenants.

De 1987 à 1999 nous avons pris l'habitude d'inviter les étudiants de première année de français à répondre anonymement par écrit à la question «Pourquoi avez-vous choisi le cours de français?». 1633 réponses ont été ainsi analysées à partir desquelles nous avons extrait 38 items récurrents qui ont servi à monter un questionnaire de 40 items $^{2}$ sur la motivation pour le cours de français. Ce questionnaire a ensuite été proposé en classe par l'auteur à 893 étudiants suivant des cours de français en option, répartis dans 16 établissements d'enseignement supérieur à Taiwan. Il est composé des six catégories de questions retenues par Candelier et Hermann-Brennecke (1993) : l'ajustement à l'environnement social, l'influence d'autres personnes, les contacts recherchés, la familiarité, l'utilité. Nous y avons ajouté une catégorie «le mieux-être» que nous avons définie de la manière suivante : elle recouvre tout ce qui est perçu par les étudiants comme affectant directement la qualité de leur vie, tout ce qui donne sens à leur existence sociale et individuelle et leur permet en même temps un retour sur leur propre culture.

La notion de motivation ne semble pas avoir fait l'objet de recherches dans le milieu des enseignants-chercheurs du français enseigné en option à Taiwan. Pour un autre type de public, celui des départements de français, il existe deux travaux (Chaubet, 1996; Monier, 1995). Leur approche s'attache à comprendre trois types de facteurs motivationnels censés leur apporter «[...] une assez bonne appréhension de notre public, tant dans ses attentes que dans la façon dont sont vécues les études de français » (Monier, 1995). Ces facteurs sont les choix de départements effectués par les étudiants avant l'examen d'entrée à l'université, l'analyse par ces derniers du contenu de leurs études et enfin leur perspective d'avenir après l'obtention de leur diplôme. Ces objectifs sont sous-tendus par des considérations pédagogiques de «remotivation» des étudiants qui sont classés en «motivés», «semi-motivés » et «non motivés », à partir du seul critère du choix du français ${ }^{3}$ pour

2. En raison du format de cet article, nous passerons sur les détails de la conception et de l'administration de ce questionnaire.

3. En fonction des résultats obtenus à la fin de leurs études secondaires, les étudiants proposent au Ministère de l'Éducation nationale trois choix de département et d'université. Celui-ci leur assigne ensuite une université et 
l'entrée à l'université. Les étudiants ayant choisi le français en premier choix sont considérés comme motivés, ceux l'ayant choisi en deuxième ou troisième choix sont les semi-motivés, tandis que ceux ne l'ayant pas choisi du tout sont les non motivés (Chaubet, 1996).

Ces études se focalisent donc sur une perspective diachronique de la motivation : celle-ci est définie à partir de la motivation avant le choix de leur discipline, de l'état présent de leur motivation (évaluation par les étudiants de leurs cours de français) et des effets sur leur motivation présente de leurs attentes professionnelles. Cependant cette notion n'est pas explicitée et elle n'est pas mise en relation avec l'environnement sociétal. Seules les représentations des étudiants du degré de difficulté/ facilité de la langue française sont mentionnées. En outre, hormis le sexe, aucune variable socioculturelle n'est utilisée pour affiner les réponses des étudiants. La position du français dans le champ de l'enseignement des langues extérieures à Taiwan, une réflexion sur les conditions d'enquête (les étudiants ont à remplir un questionnaire rédigé et analysé par leurs propres enseignants) sont également absentes. Il semble donc aléatoire de s'appuyer sur ces travaux autrement que pour rappeler des généralités telles que l'intérêt pour la culture française sous-jacente à l'apprentissage de la langue française (Chaubet, 1996).

\section{La représentation «les Français romantiques »}

Selon Jodelet (1999), la représentation sociale est «une forme de connaissance, socialement élaborée et partagée, ayant une visée pratique et concourant à la construction d'une réalité commune à un ensemble social». C'est donc une vision compacte de l'Autre, une sorte de prêt-à-porter communicatif dont la fonction cognitive consisterait à mettre en place une connaissance spontanée/naïve opposée à la rationalité du savoir scientifique. Elle est également re-présentation, dans la mesure où le groupe humain parmi lequel elle circule s'approprie ce qu'il considère comme une réalité mais qui en fait s'avère être, au moins en partie, une fiction. Cette fiction devient un trait marquant de la culture de ce groupe humain dans cette phase finale de la construction d'une représentation sociale que Roussiau et Bonardi (2001) appellent «la naturalisation». C'est ainsi qu'au Japon, à la différence du monde

un département. Ce système s'est assoupli ces dernières années mais les étudiants n'ont pas encore le libre choix de leur matière et de leur établissement. 
chinois, on ne semble pas considérer les Français comme romantiques malgré de nombreuses similitudes dans le contenu des représentations (Chi et Himeta, 2009).

Une autre caractéristique de la représentation sociale concerne son mode d'existence. Sa circulation est fluide car elle relève plus d'une information circulante proche de la rumeur que d'un savoir à transmettre. Elle se révèle donc insaisissable car elle rebondit d'individu en individu, possédant à la fois des caractéristiques facilement identifiables - le caractère romantique des Français ne semble pas devoir faire l'objet d'une explicitation dans le monde chinois - tout en se présentant sous un aspect protéiforme, ce qu'explique la théorie du centre et de la périphérie d'une représentation d'Abric (1994). Les informations reçues qui corroborent l'énoncé principal de la représentation sociale tendent à être plus facilement mémorisées et renforcent la cohésion du groupe qui fait circuler cette représentation qui possède donc une fonction identitaire. Le jugement porté sur l'Autre rassemble les membres du groupe en particulier par un effet de miroir : cet Autre est ce que nous ne sommes pas, ou du moins s'il nous ressemble, la différence reste marquée.

Ce préambule théorique permet de dépasser le discours ordinaire qui circule à Taiwan chez la plupart des enseignants de français, qu'ils soient natifs ou non, et qui présuppose que les représentations des Français doivent être évaluées selon une problématique du vrai ou du faux.

Nous avons donc entrepris de retrouver, dans la mesure du possible, le parcours de la représentation des Français romantiques qui semble constituer à Taiwan (mais également en Chine) ${ }^{4}$ une évidence ne justifiant pas d'autres précisions (Zhang, 2000; Zheng et al., 2003; Merkelbach, 2001). Comme l'affirme Zarate (1995, p. 39) «le recours à l'histoire permet de retrouver la genèse des représentations [...] le document historique permet de retrouver comment le sens s'est construit, de restaurer la diversité des intérêts qui étaient alors en jeu». Or, la Chine de la fin du XIX ${ }^{\mathrm{e}}$ siècle et du début du $\mathrm{XX}^{\mathrm{e}}$ siècle, pays en pleine crise institutionnelle et sociétale, voit fleurir de nombreuses traductions d'œuvres littéraires occidentales, notamment relevant du romantisme français, qui vont bouleverser le monde des lettres chinoises (Lee,

4. Afin d'éviter la surabondance d'informations, nous n'expliciterons pas dans cet article la problématique identitaire Taiwan/Chine que nous ferons semblant de considérer unique. 
2002). L'idée romantique de l'amour-passion va se trouver diffusée dans le grand public et devenir le noyau central de la représentation des Français romantiques. L'amour partagé va ainsi s'opposer à la conception chinoise pragmatique du mariage arrangé, thème récurrent de la littérature chinoise : «toutes les discussions sur le point de vue des Chinois sur les relations doivent prendre en compte le mariage arrangé [...]. Cette tradition prévalente a conduit à des attitudes calculées et pratiques envers les relations d'amour parmi les Chinois.» (Cho, 1995, p. 288)

D'autres éléments sont à prendre en compte dans la constitution de cette représentation. Les rapports des premiers diplomates chinois envoyés en France à partir de 1866 se révèlent très instructifs à cet égard. Ils notent la splendeur des villes en des termes dithyrambiques, en particulier Paris qui à l'époque est en pleine rénovation sous la direction du baron Haussmann : «Le soir nous avons parcouru une dizaine de $l i$ en voiture le long de larges avenues remplies de monde [...]. Les lumières de la rue sont si brillantes que la lune et les étoiles perdent tout leur éclat : c'est à vous en arracher des soupirs incessants [...]. Nulle part ailleurs il n'y a autant de jardins, théâtres, débits de boissons et maisons de thé.» (Bin Chun cité dans Lévy, 1986, p. 84.) Ces diplomates tombent sous le charme des Françaises bien habillées et respectées par leurs maris même s'ils leur trouvent des mœurs relâchées. Cette image ambigüe de la femme française perdure jusqu'à nos jours dans le monde chinois. L'image d'une belle ville où les gens savent vivre est déjà là. Pour les jeunes Chinois arrivés avec le mouvement ouvriersétudiants (Leung, 1982) dans les années 1910, l'image de la qualité de vie s'ancre définitivement dans la représentation qu'ils ont des Français. En particulier, on leur prête l'aptitude à savoir discerner et créer le beau, ce qui est reflété par le nombre considérable d'étudiants attirés par les écoles des beaux-arts (Rapport des Annales franco-chinoises, $1932)^{5}$.

Toutes les recherches convergent pour noter que de plus en plus de Taiwanais aspirent à voyager et voyagent effectivement en Europe pour leurs études (Lee, 1993) ou dans un but touristique. Le nombre

5. Il faudrait également mentionner l'influence du Japon, en particulier dans le domaine des arts pour Taiwan, et celle des États-Unis (le cinéma hollywoodien et les auteurs francophiles américains) dans l'émergence de cette représentation. Quant aux influences plus récentes, on pourrait citer le cinéma français et les séjours des étudiants taiwanais en France. 
de voyageurs à destination de la France augmente, mais il est extrêmement difficile de connaitre statistiquement leurs impressions de voyage et surtout le discours qu'ils font circuler à Taiwan sur la France et les Français. De rares enquêtes permettent au moins de connaitre leur motivation de départ, comme ces étudiants qui voyagent en Europe pour célébrer l'obtention de leur diplôme (Daxue Bao, 17/04/1998) et qui cherchent «une expérience enrichissante de la vie» à travers les beaux sites touristiques. L'ensemble des enseignants de français à Taiwan s'accordent pour citer Paris comme destination première des touristes taiwanais puis les châteaux de la Loire et la Côte d'Azur. Les sites prestigieux (Louvre, musée d'Orsay, le jardin de Monet) sont hautement prisés. Le développement sensible dans les librairies taiwanaises des rayons consacrés aux ouvrages sur les cafés de Paris, la mode, la gastronomie, l'architecture, le design d'intérieur, les arts picturaux, les jardins et le vin en France, témoigne de la circulation d'un discours qui accorde une grande place à la qualité de vie des Français.

Les Taiwanais sont de très gros consommateurs de médias audiovisuels très orientés vers les États-Unis (Lee M.-H., 1993). La part de la France est négligeable mais, pour la télévision, l'impact de la publicité est à considérer. Des publicités, généralement pour des produits haut de gamme (automobiles, résidences, bijoux), pas nécessairement français, s'appuient sur des éléments de la culture française (chansons, paysages, noms de lieux célèbres, personnages historiques) qui jouent systématiquement sur les notions de prestige et de raffinement. Mais c'est dans les médias écrits spécialisés, comme les revues de mode ou de design, que la France est la plus présente.

C'est enfin sur l'édition francophile que nous nous sommes appuyés pour cerner l'ancrage de la représentation des Français romantiques dans la société de Taiwan, car comme l'affirme Umberto Eco (2000, p. 27) :

Curieusement, nous voyageons grâce à notre connaissance antérieure de ce que nous sommes sur le point de découvrir, puisque des livres déjà lus nous ont indiqué ce que nous étions censés trouver. L'influence de ces livres de référence est telle que les voyageurs, quelles que soient leurs découvertes et les réalités perçues, vont tout interpréter et tout expliquer en fonction de ces ouvrages.

Cette importance du livre nous a amené à choisir sept ouvrages taiwanais de grande diffusion qui véhiculent des représentations de la France et des Français. Ils confirment à la fin du $\mathrm{Xx}^{\mathrm{e}}$ siècle, avec quelques variantes peu significatives, la centralité des «Français romantiques » 
dans l'ensemble des représentations qu'ont les Taiwanais à propos des Français.

Nous avons également complété cette analyse de discours par des entretiens avec des Taiwanais francophones et des résidents français installés depuis longtemps sur l'ile, ce qui nous a permis de vérifier les conclusions de nos recherches.

\section{Évolutions sociales et identitaires}

Pour comprendre comment cette représentation influence la motivation de nos étudiants pour le choix des cours de français, il faut noter le rôle de l'évolution politico-économique de Taiwan depuis les années 1980. C'est en 1987 que la loi martiale a été abolie à Taiwan et qu'un régime démocratique s'est progressivement installé dans le pays. Parallèlement, le développement économique s'est accru exponentiellement durant ces années. Il en résulte deux faits extrêmement importants pour comprendre notre propos. Les voyages à l'étranger sont devenus libres et accessibles pour tout le monde et le nouveau pouvoir économique des Taiwanais leur a permis une consommation débridée (Guiheux, 2000) de biens tangibles (grandes marques de vêtements, vins, cosmétiques) et intangibles (loisirs, voyages à l'étranger). Les enfants de cette génération sont donc nés dans une relative aisance, ce qui leur a rendu plus accessible cette qualité de vie qu'ils prêtent aux Français. La fréquentation assidue des cafés à Taiwan en est un symbole évident.

Or les générations actuelles d'étudiants se voient attribuer par le monde adulte dominant, dont ils dépendent matériellement, des valeurs, des attitudes et des comportements à connotation négative (Yang, 1999). Ainsi, l'étiquette fourre-tout de «génération des fraises » utilisée pour décrire cette jeunesse insinue un manque de résistance à la pression du travail. En fait, ces générations se caractérisent par leur plus grande ouverture au monde. Parallèlement, elles assistent à un renouvellement de la tradition à Taiwan. En fait, deux traditions sont en compétition : une tradition chinoise, héritage du gouvernement nationaliste d'avant 1987, et une tradition taiwanaise, concept qui a émergé progressivement depuis 1987. Ce sont deux constructions politiques qui s'opposent mais qui ne proposent, ni l'une ni l'autre, de réponse complète aux évolutions actuelles de la société taiwanaise que connaissent les étudiants. La mondialisation, dont les conséquences politiques et économiques sont sensibles à Taiwan, surtout pour les jeunes générations, leur offre une alternative aux conflits identitaires de leurs ainés. Ils conservent 
certains traits de la société traditionnelle, qu'elle soit chinoise ou taiwanaise, mais l'occidentalisation avancée de Taiwan les amène à développer des valeurs plus individualistes, et à afficher des comportements envers les études (le travail à temps partiel) qui bousculent les processus de socialisation au sein de la famille et des réseaux de clientèle. De surcroit, la situation politico-économique de Taiwan leur ouvre des horizons professionnels (le marché européen et de Chine Populaire) qui étaient inaccessibles pour leurs parents. Ces phénomènes engendrent un renouvellement identitaire pour ces générations d'étudiants. En ce qui concerne les étudiants de français, leur intérêt pour les dimensions culturelles de l'enseignement-apprentissage du français s'inscrit dans cette évolution. Il procède d'une valeur sociale que nous nommerons désirabilité de l'étranger, caractéristique de ces nouvelles générations, valeur qui ne possédait pas de terrain d'expression pour leurs parents.

\section{La motivation existentielle}

Nuttin (1996), dans le modèle dit relationnel, définit la motivation non comme un processus de déclenchement mais comme «[...] l'orientation dynamique continue qui règle le fonctionnement, également continu, de l'individu en interaction avec son milieu». Ces interactions répondent aux besoins des individus qui vont agir en vue d'un objet-but, en passant éventuellement par ce que Nuttin appelle des objets-moyens. Ce sont des étapes nécessaires pour atteindre un but, comme suivre une école préparatoire pour passer avec succès l'examen d'entrée dans une grande école puis finalement obtenir le diplôme convoité. Le besoin défini comme «une relation "requise" entre l'individu et le monde» est un élément de première importance dans cette théorie. Les interactions peuvent se présenter sous un angle négatif, par des insuffisances fonctionnelles qui génèrent un besoin de compensation. De manière positive, elles se présentent notamment sous la forme de renforcements affectifs. Ce ne sont donc plus les besoins ni les contraintes et stimuli qui doivent attirer principalement l'attention pour comprendre la motivation humaine mais plutôt le jeu des interactions individu-monde, dans ce qu'il a de répétitif et de repérable. La motivation ne se trouve plus enfermée dans le psychisme individuel mais s'ouvre sur le monde. L'individu devient «un sujet en situation»; aussi l'explication de la motivation ne se résume pas à un choix entre la prépondérance de l'individu ou de l'environnement. Ces deux pôles s'impliquent l'un l'autre. En attribuant un rôle important, en particulier aux représentations sociales 
des étudiants de Taiwan, mais également à leurs valeurs sociales et à la question identitaire, nos recherches s'inscrivent dans cette perspective organique de la motivation.

Quant à la théorie de Gardner et Lambert (1972) de la motivation intégrative et instrumentale qui est l'objet d'un assez large consensus parmi les enseignants-chercheurs de Taiwan, les résultats de notre enquête montrent qu'elle n'est guère opératoire pour le français enseigné en optionnel. En effet, la grande majorité des apprenants de français ont répondu ne pas avoir comme projet d'étudier dans un pays francophone ou d'y séjourner. La seule réponse qui pourrait s'apparenter à la motivation intégrative concerne l'envie de voyager en France, mais qui est exprimée sous la forme d'un désir et non pas d'un projet qui justifierait l'apprentissage du français.

Notre hypothèse est donc que la motivation des étudiants de Taiwan s'appuie sur un intérêt marqué pour la culture française, englobant $\mathrm{cul}$ ture cultivée et culture de masse (Kalifa, 2001). Cet intérêt est reflété en particulier par la représentation « les Français romantiques » qui circule dans toutes les couches de la population à Taiwan. Mais cette culture, dont la connaissance procède en partie du désir de construire un savoir encyclopédique sur la France et les Français, serait surtout pour les étudiants un objet-moyen, selon la conception de J. Nuttin. Selon nous, elle permettrait d'atteindre un objet-but qui serait l'amélioration qualitative de leur existence en tant qu'individus, point sur lequel la société taiwanaise resterait insatisfaisante aux yeux de la jeune génération. C'est en ce sens qu'on évoque une motivation existentielle dont les principaux traits peuvent s'énoncer comme suit.

Une motivation existentielle peut s'accommoder d'orientations instrumentales et intégratives. Les buts de l'apprenant qui consistent à améliorer sa qualité de vie et à donner plus de sens à son existence sociale et individuelle entrainent une orientation qui rompt avec les orientations instrumentales et intégratives. Un étudiant de français à Taiwan, par exemple, peut choisir cette langue pour voyager en France, affirmer son statut social, orientations instrumentales qui rejoignent un véritable intérêt pour le rôle de l'art en France (orientation intégrative). Ces motivations ne sont cependant que des étapes pour atteindre un autre but qui serait l'introduction d'un mode de vie plus romantique dans le quotidien de la société taiwanaise, jugé trop pragmatique par l'apprenant (motivation existentielle).

Les apprenants de français à Taiwan sont très attirés par la culture française mais en raison de leur habitus d'apprentissage de l'anglais, ils 
éprouvent des difficultés à formuler cet intérêt dans un cours de langue étrangère. Les réponses du type «on est intéressé par la langue» masquent souvent cet intérêt pour les dimensions culturelles qui n'est pas légitimé dans les cours de langue étrangère à Taiwan. La motivation pour une langue devient ainsi, elle aussi, un objet-moyen, une étape vers la découverte de la culture d'un pays. La motivation existentielle du choix d'une langue étrangère peut donc se présenter sous la forme trompeuse d'un prétexte à faire autre chose, mais qui ne s'inscrit pas dans une tradition éducative. L'étendue de son domaine englobe tous les niveaux de l'existence mais privilégie cependant l'affectif, les représentations de soi et de l'autre jouant un rôle fondamental ainsi que les valeurs sociales de l'apprenant.

Par rapport au paradigme de R. Gardner et de W. Lambert insistant sur les risques d'acculturation, la motivation existentielle affecte le rôle de l'autre (le groupe, l'étranger) qui n'agit plus alors sur l'apprenant comme une force centrifuge à travers diverses formes d'assimilation ou d'intégration. Elle tend au contraire à devenir une force centripète en incitant l'apprenant à se centrer sur son groupe d'appartenance, les apports de la langue-culture étrangère étudiée devenant une (res)source du développement personnel.

Cette inversion des rôles peut affleurer à la conscience des apprenants - certains étudiants choisissent délibérément d'intégrer les influences étrangères pour enrichir leur vécu (ou elles se produisent à leur insu) - mais nombre d'entre eux n'ont pas entrepris de réflexion sur leur relation avec l'étranger. Comme le rappelle Nuttin (1996), il n'est plus question de donner la prépondérance à l'individu conscient ou non de sa motivation ou à l'influence de l'environnement. Tout se joue en effet dans sa relation avec le monde, imbriquant les niveaux biologique, affectif, social et culturel.

\section{Les motifs du choix du cours de français à Taiwan}

L'enquête qui a servi de support à cette recherche est principalement divisée en quatre parties : les données biographiques (15 items), les motifs du choix du cours de français (40 items), la valeur sociale que nous avons appelée «désirabilité de l'étranger» (86 items). En ce qui concerne les données biographiques, nous avons inclus des questions sur l'ethnie des parents qui tiennent compte de la situation sociale et politique de Taiwan. Elles n'ont pas généré d'informations particulièrement signifiantes à propos des deux ethnies principales, les Minnans 
et les Continentaux, au contraire de la profession des parents dont l'influence était attendue. Quant aux questions sur le projet d'aller étudier à l'étranger, moins de $10 \%$ des réponses sont positives et elles ne concernent pas, pour la plupart, un pays francophone. Pour ce qui est de la profession envisagée, on constate une forte proportion de réponses qui n'ont rien à voir avec la discipline suivie par les étudiants, ce qui traduit clairement le malaise de la population étudiante taiwanaise. Il serait intéressant, dans le cadre d'une autre recherche, d'approfondir le lien direct entre le choix du cours de français et le désintérêt des apprenants pour leurs études actuelles.

Les réponses aux questions sur le motif du choix du cours de français sont réparties en très d'accord (A), d'accord (B), pas d'accord (C), pas du tout d'accord $(D)$, sans avis $(E)$. On notera que les questions portent sur le choix du cours de français et non pas sur le choix de la langue française. Cette distinction est justifiée par notre connaissance de la culture éducative locale qui amène souvent les étudiants taiwanais à choisir des cours en option pour des motifs autres qu'académiques et non pour l'intérêt du contenu. On retiendra en particulier l'importance de la représentation de la langue française mélodieuse $(A+B=78,7 \%)$ et son utilité pour la promotion professionnelle $(A+B=61,5 \%)$. On notera que le discours circulant dans la société taiwanaise valorise avec insistance l'apprentissage de plusieurs langues étrangères pour la carrière, ce qui motive le haut pourcentage des réponses favorables des apprenants malgré le manque d'informations objectives sur ce domaine. L'intérêt pour la culture française génère plusieurs pourcentages favorables très élevés : $68 \%$ de réponses mentionnent le choix du cours pour comprendre la qualité de vie des Français; 78,4\% espèrent recevoir beaucoup d'informations sur la France; 75,5\% ont entendu dire que la France est un pays romantique, etc. On constate donc que les représentations de la langue continuent à jouer un rôle non négligeable mais que l'importance des dimensions culturelles dans le choix du cours devrait amener le corps enseignant à Taiwan à réviser ses stratégies (la plupart des enseignants interrogés n'accordent qu'un rôle ludique et bouchetrous à la culture dans leurs cours).

Les questions sur la désirabilité de l'étranger sont réparties en quatre parties: 1. Attitudes des étudiants envers les étrangers de Taiwan. 2. Étudiants ayant une expérience de séjour ou de voyage à l'étranger. 3. Comment les étudiants perçoivent les Français et les Américains à travers les médias. 4. Si vous viviez à l'étranger. Ces catégories de questions ont pour but d'analyser avec le plus de finesse 
possible les représentations des étrangers, en particulier les Occidentaux, et l'expérience des apprenants de ces étrangers. On espère ainsi comprendre comment cette valeur sociale, constatée pour toute la population à Taiwan et que nous avons appelée désirabilité de l'étranger, influence le choix du cours de français par les apprenants. Toutes les réponses proposées prennent en compte les réalités locales et ont fait l'objet d'une pré-enquête expérimentale.

1. En ce qui concerne les attitudes des étudiants, on constate succinctement que 1,5\% des apprenants considèrent que les Européens ont une influence néfaste sur la société taiwanaise, que 99,3\% pensent pouvoir faire confiance aux Européens et Américains de Taiwan, que $78,5 \%$ les trouvent pleins d'assurance tandis que $74,8 \%$ les considèrent ouverts d'esprit. On notera aussi que $66,7 \%$ les trouvent romantiques tout en sachant qu'en général seuls les Français sont l'objet de cette représentation. D'ailleurs une majorité de réponses définit être romantique comme faire ce que vous avez envie sans but précis, l'art joue un rôle important dans votre vie, exprimer librement ses sentiments sans tenir compte de l'opinion des autres gens, l'amour est le plus important dans la vie (seulement 4,7\% des réponses concernent d'autres aspects). Il apparait donc clairement que cette valeur constitue un facteur très favorable au choix du cours de français.

2. Quant à leur expérience de l'étranger, $49 \%$ d'entre eux ont voyagé dans d'autres pays et parmi ceux-ci $48,4 \%$ ont envie de recommencer l'expérience, en grande partie en raison de la découverte de la diversité culturelle et pour l'aspect épanouissement personnel. Là encore, le contexte se présente favorablement pour les choix des cours de français.

3. Cette catégorie insiste sur le rôle des médias qui restent la source principale des représentations des Français. Le cinéma français leur laisse l'impression que les Français savent profiter de la vie $(79,1 \%)$, qu'ils sont passionnés d'art (54,3\%), qu'ils sont sensibles et raffinés $(49,3 \%)$ et qu'ils sont trop imbus de leur culture $(43,1 \%)$ pour les principaux résultats. Ce cinéma leur permet d'apprécier une manière plus libre et décontractée d'expérimenter la sensualité $(51,8 \%)$ tandis que $46,5 \%$ considèrent apprendre une autre manière d'exprimer leurs sentiments. On notera également que 59,4\% trouvent les Français romantiques dans les films. Toutes ces réponses diffèrent très sensiblement des réponses pour les Américains. On retiendra également l'influence de la lecture mais surtout les revues de mode, la population des classes de français étant en grande majorité féminine. 
4. Dans cette partie du questionnaire, on a surtout cherché à mettre en valeur la dimension fantasmatique du choix des cours de français. On retient en particulier qu'après avoir vécu à l'étranger 59,3\% d'entre eux s'imaginent plus curieux du monde; $80,3 \%$ plus forts mentalement; $69 \%$ seraient plus à l'aise pour socialiser avec autrui; 48,1\% deviendraient plus individualistes. Ils aimeraient que leur entourage remarque à leur retour leur nouvelle expérience de la vie $(74,3 \%)$, leur maitrise des langues étrangères (71\%), leur connaissance des cultures étrangères $(70,1 \%)$. Ils sont $76,9 \%$ qui aimeraient vivre à l'étranger pour faire ce dont ils ont envie et $76,1 \%$ qui souhaiteraient ramener de nouvelles attitudes de vie à Taiwan tandis que $54,1 \%$ sont convaincus qu'ils gagneraient confiance en eux pour exprimer leurs sentiments et leur manière de penser sans avoir à suivre aveuglément l'avis du groupe. On discerne dans l'ensemble des réponses un grand désir d'émancipation de la part des apprenants. Il nous a conduit à associer le choix du cours de français à un processus de construction identitaire et par voie de conséquence à une motivation existentielle.

On rappellera enfin que toutes les questions sont traduites en chinois et soumises au préalable à une relecture de collègues francophones et sinophones. Par ailleurs, les statistiques appliquées au questionnaire à partir du prologiciel SPSS de Pearson dans lequel les valeurs de référence pertinentes sont calculées à partir du système du khi carré, montrent des résultats très satisfaisants.

\section{Conclusion}

Sur le plan didactique, la prise en compte de la motivation existentielle permet aux enseignants d'affiner leur compréhension du public et d'affiner leur stratégie d'enseignement. Ils peuvent ainsi mieux répondre aux véritables besoins des apprenants, à condition d'analyser extensivement et finement la situation éducative. Dans ce type de démarche, les tâches sont nombreuses, voire complexes, car elles concernent non seulement des besoins mais aussi les rêves des apprenants : " [Il faudrait] construire, en meilleure connaissance de cause, d'autres démarches d'enseignement, qui soient adaptées [...] aux attentes et aux rêves d'ailleurs des apprenants, dans des équilibres fugitifs d'autant plus passionnants à rechercher.» (Beacco, 2000, p. 13.) L'enseignant ne peut plus dès lors se contenter des réponses des apprenants du type «j'apprends le français parce que ça m'intéresse » pour fonder sa stratégie d'enseignement. Il ne s'agit évidemment pas d'abandonner l'enseigne- 
ment de la langue au profit des dimensions culturelles mais de donner à ces dernières la place qui leur revient. Cela implique aussi que l'enseignement des aspects culturels soit pensé non pas dans le style carte postale que l'on trouve dans nombre de méthodes à prétention universaliste, mais qu'il fasse un large appel aux sciences sociales (Beacco, 2000).

Enfin, on peut réfléchir, en termes de politique linguistique, à l'intérêt de mieux mettre en valeur la francophilie dans des régions du monde comme l'Asie où le capital affectif pour la France reste important malgré le déclin de l'apprentissage du français.

\section{RÉFÉRENCES BIBLIOGRAPHIQUES}

ABric J.-C. (1994) : «L'organisation interne des représentations sociales : système central et système périphérique», dans C. Guimelli, Structures et transformations des représentations sociales, Neufchâtel, Delachaux et Niestlé, p. 73-84.

Beacco J.-C. (2000) : Les dimensions culturelles des enseignements de langue, Paris, Hachette.

BeAcco J.-C. (2009) : «Instruments de référence pour l'enseignement des langues/du français et diversité des cultures éducatives », dans $L e$ français, la francophonie et la francophilie en Asie-Pacifique, Paris, L'Harmattan.

Boк S.-G. (2004) : «L'enseignement du français et la Corée», dans H. Kato, La modernité française dans l'Asie littéraire, Paris, Presses Universitaires de France, p. 295-301.

Candelier M. et Hermann-Brennecke G. (1993) : Entre le choix et l'abandon : les langues étrangères à l'école, vues d'Allemagne et de France, Paris, Crédif/Didier.

Chaubet P. (1996) : Analyse des motivations et attentes d'étudiants chinois apprenant le français. Le cas de l'université Fujen à Taiwan, Taipei, Chung Yang.

Chi H.-P. et Himeta M. (2009) : «Représentation de la France chez les étudiants taiwanais et japonais », dans Le français, la francophonie et la francophilie en Asie-Pacifique, Paris, L'Harmattan, p. 187-199.

Сно W. (1995): Taiwanese love styles and their association with selfesteem and relationship quality, Taipei, Genetic, social, and general psychology monographs.

Daxue bao, article de presse du 17/04/1998, Taipei, Taiwan, Éd. Université Chengchi. 
DREYER S. (2005) : «Apprenants de Taiwan de langues étrangères et enseignants occidentaux, une relation complexe», dans J.-C. Beacco et al., Les cultures éducatives et linguistiques dans l'enseignement des langues, Paris, Presses Universitaires de France, p. 225-244.

Eco U. (2000) : Ils cherchaient des licornes, Paris, Alliage, $\mathrm{n}^{\circ}$ 41-42.

Gardner R. et LAmbert W. (1972) : Attitudes and motivation in second language learning, Rowley, Newbury House.

Guineux G. (2000) : «Consommation et société : l'enrichissement matériel comme fin en soi ?», dans C. Chaigne et al., Taiwan, enquête sur une identité, Paris, Karthala, p. 237-248.

Jodelet D. (1999) : Les représentations sociales, Paris, Presses Universitaires de France.

Kalifa D. (2001) : La culture de masse en France, 1860-1930, Paris, La Découverte.

LeE G. (2002) : La Chine et le spectre de l'Occident, Contestation poétique, modernité et métissage, Paris, Syllepse.

LEE M.-H. (1993) : The effects of American media on image of the United States and value formation in Taiwan, thèse de doctorat, State University of New York, États-Unis.

LeUnG K.-C. (1982) : The Chinese work-study movement: the social and political experience of Chinese students and students-works in France 1913-1925, thèse de doctorat, Brown University, États-Unis.

LÉvy A. (1986) : Nouvelles lettres édifiantes et curieuses d'ExtrêmeOccident par des voyageurs lettrés chinois à la Belle Époque, Paris, Seghers.

Merkelbach C. (2001): Conceptualization of foreign language teaching as an intercultural task based on the perception of foreigners by Taiwanese youth, Taipei, NTU Studies in Language and Literature.

Monier A. (1995) : Étudier le français. Pourquoi? Comment? Étude sur les motivations et les attentes des étudiants du département de français, Taipei, Université Tamkang.

Nuttin J. (1996) : Théorie de la motivation humaine. Psychologie d'aujourd'hui, Paris, Presses Universitaires de France.

Roussiau N. et Bonardi C. (2001) : Les représentations sociales. État des lieux et perspectives, Sprimont, Mardaga.

TANG X. (2004) : «L'enseignement du français langue étrangère en Chine : histoire, problèmes et perspectives », dans H. Kato, La modernité française dans l'Asie littéraire, Paris, Presses Universitaires de France, p. 283-294.

YANG Z. (1999) : Taiwan dreamer, Taipei, Xin Xin Wen, p. 283-294.

ZaRATE G. (1995) : Représentations de l'étranger et didactique des langues, ENS Fontenay/Saint Cloud, Didier. 
ZHANG C. (2000) : L'enseignement/apprentissage de la langue française auprès des étudiants chinois, études de leurs représentations, mémoire de DEA, Université Paris 7.

Zheng L.-H., Desjeux D. et Boisard S. (2003) : Comment les Chinois voient les Européens?, Paris, Presses Universitaires de France. 